\title{
Los desafíos del futuro: crecimiento poblacional y desarrollo
}

\section{María Emilia Burgos ${ }^{1}$}

\section{Contribución en la Sección Política Internacional}

\section{Introducción}

Mientras en la actualidad se cuenta con una población mundial de 7.300 millones, para el año 2100 se proyecta un crecimiento que sume alrededor de 4 mil millones de personas y tales proyecciones permiten avizorar los problemas de un futuro inmediato.

Existen diferentes factores que a lo largo del siglo pasado han ido impactando y produciendo cambios en la población mundial, relacionados a diversos acontecimientos globales, políticas migratorias, guerras, desastres naturales, flujo de personas, etc. Esas diversas dinámicas sociales se aprecian en ejemplos tales como la disparidad de género que, de acuerdo al informe del United Nations Population Fund/ UNFPA (2017), podría socavar la paz y los objetivos del desarrollo en marcha. En tal sentido, sólo alrededor de la mitad de las mujeres del mundo tienen empleos remunerados, ganando $77 \%$ de aquello que los hombres obtienen. En ese marco, 3 de cada 5 mujeres en el mundo carecen de licencia por maternidad.

El informe señala que la población mundial aumentará a casi el doble en 100 años, existiendo en la actualidad situaciones dispares en las diversas regiones del planeta (disminución poblacional por un lado y en contraste, otros puntos del mundo muestran una población en alza, con grandes deficiencias de infraestructura).

De acuerdo a United Nations/UN Department of Economic and Social Affairs (2017), se espera que la población mundial, que actualmente es de 7.6 mil millones, alcance los 8.6 mil millones en 2030, 9.8 mil millones en 2050 y 11.2 mil millones en 2100, incorporándose aproximadamente 83 millones de personas a la población del planeta cada año, con una tendencia ascendente en el volumen poblacional, aun cuando continuaran disminuyendo los niveles de fertilidad.

Las proyecciones poblacionales se basan en determinadas asunciones, tales como la de la declinación de la fertilidad en países donde las familias numerosas son aún comunes como un pequeño crecimiento de la misma en países en los que las mujeres tienen 2 hijos como promedio, a lo largo de sus vidas. Se proyecta el aumento de tasas de supervivencia en todos los países, así como la declinación de las tasas de mortalidad en todos los rangos etarios.

\footnotetext{
1 Estudiante de Relaciones Internacionales (Pontificia Universidad Católica Argentina, Paraná, Entre Ríos, República Argentina) y miembro de Equilibrium Global.
} 
El aumento poblacional que se prevé no será uniforme. Más de la mitad del crecimiento poblacional previsto entre el presente y 2050 se estima que ocurrirá en África, siendo Asia el segundo mayor contribuyente al crecimiento poblacional; seguidos ambos por América Latina, América del Norte y Oceanía, con un crecimiento más modesto. Por su parte, Europa aparece proyectado como el único espacio con una población más pequeña en 2050 de la que poseía en 2017.

Así, el crecimiento de la población continuará siendo especialmente alto en el grupo de 47 países designados por Naciones Unidas como los países menos desarrollados, entre los cuales están incluidos 33 países africanos. Al mismo tiempo y en marcado contraste con ello, se estima que las poblaciones de otros 51 países disminuirán entre 2017 y 2050 (en países como Bulgaria, Croacia, Letonia, Lituania, Polonia, entre otros, se espera que su población disminuirá más del $15 \%$ hacia 2050) (UN Department of Economic and Social Affairs, 2017).

En el mismo período, se espera que la mitad del crecimiento de la población mundial se concentre en sólo 9 países: India, Nigeria, República Democrática del Congo, Pakistán, Etiopía, la República Unida de Tanzania, Estados Unidos de América, Uganda e Indonesia (ordenados por su contribución esperada al crecimiento total). Por lo demás, las proyecciones indican que, en aproximadamente 7 años, la población de la India superará a la de China, esperándose que ambos países posean aproximadamente 1440 millones de personas hacia 2024 y que a partir de ese momento, la población india continúe creciendo durante varias décadas a alrededor de 1.5 mil millones en 2030 y acercándose a 1.66 mil millones en 2050, mientras que la población china permanezca estable hasta la década de 2030, tras lo que comenzará un lento declive.

Entre los 10 países más grandes del mundo, 1 se encuentra en África (Nigeria), 5 en Asia (Bangladesh, China, India, Indonesia y Pakistán), 2 en América Latina (Brasil y México), 1 en América del norte (Estados Unidos) y 1 en Europa (Federación Rusa). En ese marco, se proyecta que Nigeria se convierta en el tercer país más grande del mundo. Se espera una gran variación en los niveles de fertilidad entre países y regiones.

A nivel mundial, la esperanza de vida al nacer aumentó en 3,6 años entre 2000-2005 y 2010-2015 (o el paso de 67.2 a 70.8 años), observándose un aumento en todas las regiones, aunque África fue aquella en la que se observan las mayores ganancias. Se espera que globalmente, la esperanza de vida ascienda de 71 años en 2010-2015 a 77 años en 2045-2050, con diferencias regionales (UN Department of Economic and Social Affairs, 2017). 


\section{Desproporciones poblacionales, territoriales y de peso económico}

Los países más grandes del mundo en términos de población son en la actualidad China e India, ambos con poblaciones mayores a 1000 millones. Estados Unidos ocupa el tercer lugar con 325 millones de personas. Los países BRIC (Brasil, Rusia, India y China), considerados como las cuatro principales economías emergentes que se espera dominen el siglo XXI, se encuentran entre los 10 países más poblados, lo que se relaciona a su vez con su expansión económica.

Según señala el Fondo Monetario Internacional, varios países considerados en desarrollo (es decir, que no han alcanzado un alto grado de industrialización en relación con sus poblaciones y en lo que la población típicamente tiene un nivel de vida medio a bajo), también poseen considerables poblaciones (como Nigeria, con más de 190 millones; Bangladesh, con casi 165 millones y México, con alrededor de 129 millones).

En comparación con algunas de las economías más grandes del mundo, que poseen poblaciones más pequeñas (de menos de 100 millones de habitantes), particularmente en Europa, países como el Reino Unido, Alemania, Francia e Italia se encuentran entre las 10 economías más importantes.

Asimismo, varios países extremadamente pequeños, como Mónaco, Luxemburgo y las Islas Caimán, sólo cuentan con 1 millón de habitantes, con un rol más importante en el mundo financiero de lo que el volumen de su población permitiría suponer.

Por otro lado, Canadá, muy importante actor económico y uno de los países más grandes del mundo por su masa territorial, posee una población relativamente pequeña para su tamaño, con alrededor de 36.5 millones de habitantes.

\section{Urgencia de sostenibilidad}

Ante tal panorama y atendiendo a los desafíos inmediatos, puede tomarse como "hoja de Ruta" a los Objetivos de Desarrollo Sostenible (ODS), por el peso de su propuesta colectiva, que suceden a los denominados Objetivos de Desarrollo del Milenio.

En 2015, 193 estados miembros de las Naciones aprobaron la Agenda 2030 para el Desarrollo Sostenible, definiendo así los 17 ODS, con sus 169 metas. Tomando en cuenta el planteo de que vivimos en una "era urbana", en tanto por primera vez en la historia de la especie humana, supuestamente más de la mitad de población del mundo vive en ciudades (Brenner Schmid, 2016), y los planteos de Ascher (2007) acerca de la denominada "tercera revolución urbana moderna", cabe señalar que el Objetivo 11 establece: "lograr que las ciudades y los asentamientos humanos sean inclusivos, seguros, resilientes y sostenibles". 
En ese marco, se estima que el 95\% de la expansión urbana alcanzará a los países en desarrollo, lo que aumentará la vulnerabilidad frente al cambio climático y los desastres naturales. Por ello, se considera que el crecimiento de las ciudades debe ser acompañado conjuntamente de un desarrollo sostenible y de la seguridad humana, que permita pensar en sujetos a ser asegurados frente a amenazas tales como la contaminación del aire, el cambio climático, la pérdida de espacio verde y biodiversidad, etc., a través de políticas públicas eficientes que generen una mejor planificación y gestión urbana.

Como señala el United Nations/UN System Task Team on the Post-2015 UN Development Agenda (2012), el cambio acontecido en el hábitat dominante de la población mundial hace del proceso de urbanización una de las tendencias globales más significativas del siglo XXI. Las áreas urbanas no funcionan solamente como espacios de asentamiento, producción y servicios, sino que influyen en las relaciones sociales y políticas a todos los niveles, determinando avances y contratiempos en diversos ámbitos y aportando nuevos contenidos, constituyéndose en centros de poder, que influyen también en cuestiones ambientales y procesos de sostenibilidad.

Se estima que hacia 2030, el número de personas que habita en ciudades aumentará hacia 5000 millones. Será necesario, por lo tanto, buscar soluciones y estrategias que permitan a la población vivir en ciudades más seguras, resilientes y sostenibles, con una gestión que implique la cooperación entre los gobiernos, el sector privado, las ONG's y el público.

Desde la perspectiva de urbanización mundial de las Naciones Unidas, la "urbanización sostenible" resulta clave para un desarrollo exitoso de las poblaciones. A medida que el mundo continúa urbanizándose y creciendo, el desarrollo sostenible depende cada vez más de cuán exitosa sea la gestión urbana, sumada a las políticas públicas integradas con el objetivo de mejorar las vidas de los habitantes de zonas urbanas y rurales.

Las tres dimensiones del desarrollo sostenible: económico, social y ambiental se encuentran en estrecha relación con el crecimiento urbano. Una urbanización bien gestionada, con tendencias a largo plazo, puede contribuir a maximizar los beneficios y minimizar el deterioro ambiental y otras externalidades negativas.

Aunque algunos consideran que resulta costoso poner en práctica acciones sostenibles, debe tenerse en cuenta que tales costos son mínimos en comparación a los múltiples beneficios que se puede obtener, fundamentalmente facilitando la satisfacción de nuestras necesidades sin comprometer a las generaciones futuras, como señalaba en Informe Brundtland (Report of the World Commission on Environment and Development: Our Common Future, 1987). 


\section{Trayectorias de la urbanización}

La División de Población del Departamento de Asuntos Económicos y Sociales de las Naciones Unidas ha elaborado desde 1988 estimaciones y proyecciones revisadas sobre las poblaciones urbanas y rurales de todos los países del mundo y de sus principales aglomeraciones urbanas. Las Perspectivas de la urbanización mundial se utilizan ampliamente en las Naciones Unidas y en muchas organizaciones internacionales, centros de investigación, investigadores académicos y medios de comunicación.

La edición 2018 de tales Perspectivas señala que 55\% de la población mundial reside en zonas urbanas y se proyecta que para el $2050,68 \%$ de la población mundial lo hará. Las regiones más urbanizadas incluyen a América del Norte con $82 \%$, América Latina y el Caribe con $81 \%$, Europa con $74 \%$ y Oceanía con $68 \%$. El nivel de urbanización en Asia en este momento, se aproxima al 50\%. Por el contrario, África sigue siendo principalmente rural, con sólo el $43 \%$ de su población viviendo en áreas urbanas.

Desde 1950, la población rural mundial ha crecido lentamente y se espera que alcance su punto máximo en los próximos años. Actualmente se acerca a los 3.4 mil millones de habitantes y se espera que aumente un poco más, pero irá disminuyendo hacia 2050, con alrededor de 3.1 mil millones. África y Asia tienen el 90\% de población rural, siendo la India el país con mayor población rural (893 millones de personas), seguida de China, con 578 millones de personas.

Asimismo, la población urbana del mundo ha crecido rápidamente desde 1950, pasando de 751 millones a 4.2 mil millones en 2018.

Puesto que el crecimiento en la población urbana está impulsado por un lado por el aumento general de la población y por otro por el cambio al alza en el porcentaje que vive en áreas urbanas, se prevé que estos dos factores agreguen 2.5 mil millones a la población urbana del mundo hacia 2050, con casi $90 \%$ de este crecimiento sólo en Asia y África.

India, China y Nigeria, sumarán el 35\% del crecimiento de la población urbana del mundo, entre 2018 y 2050. India, con 416 millones de habitantes urbanos; China, con 255 millones y Nigeria, con 189 millones. No obstante, cerca de la mitad de los habitantes urbanos del mundo residen en asentamientos con menos de 500.000 habitantes, mientras que 1 de cada 8 vive en 33 megaciudades con más de 10 millones de habitantes. Se prevé que hacia 2030, el mundo contará con 43 megaciudades, la mayoría de ellas, en países en desarrollo.

Actualmente, Tokio es la ciudad más grande del mundo, con una aglomeración de 37 millones de habitantes, seguida de Nueva Delhi con 29 millones, Shanghai con 26 millones, Ciudad de México y São Paulo con alrededor de 22 millones de habitantes, etc. Por su parte, El Cairo, Mumbai, Beijing y Dhaka tienen cerca de 20 millones de habitantes cada una. 
Otro elemento a tener en cuenta, siguiendo la Agenda 2030 para el Desarrollo Sostenible, es la migración internacional, a la que dicha agenda reconoce como una fuerza positiva para el desarrollo económico y social, ofreciendo un mecanismo para reequilibrar los mercados laborales, siendo los mayores receptores de migrantes Europa, América del Norte y Oceanía y los mayores contribuyentes a los flujos migratorios, África, Asia, América Latina y el Caribe (UN Department of Economic and Social Affairs, 2017).

\section{Nota Final}

Tomando en cuenta todo lo señalado, a modo de cierre, cabe deslindar el escenario dado por los elementos mencionados, que hacen a la vida actual de la población mundial: ascenso de la tasa de crecimiento de la población, urbanización creciente y múltiples desafíos inmediatos, tienen como una de sus respuestas, a la sostenibilidad ambiental urbana.

Dicha sostenibilidad aparece como un sistema de incidencia a escala mundial, que no diferencia los límites político - administrativos de los distintos países, sino que por el contrario se plantea como un imperativo para todos.

Es allí donde la consideración del aporte de los países de altos ingresos debiera aumentar para hacer una verdadera diferencia en las condiciones de todas las ciudades, contribuyendo a evitar desequilibrios que influyan en la contaminación, daño a recursos naturales, movimientos poblacionales, etc. Tal contribución al desarrollo local tendría seguramente un impacto en el desarrollo global, en la búsqueda de nuevos equilibrios y en la reafirmación del soporte ético de la consideración de tales cuestiones.

\section{Referencias}

Ascher, F. (2007). Los Nuevos Principios del Urbanismo. El fin de las ciudades no está a la orden del día. Madrid: Alianza Editorial.

Brenner, N. y Schmid, Ch. (2016). La "era urbana" en debate. EURE, 42 (127), 307-339. Recuperado de: https://scielo.conicyt.cl/pdf/eure/v42n127/art13.pdf

Report of the World Commission on Environment and Development: Our Common Future (1987). Oxford: Oxford University Press. Recuperado de: http://www.undocuments.net/our-common-future.pdf

United Nations Population Fund/UNFPA (2017). Estado de la población mundial 2017: Mundos aparte: la salud y los derechos reproductivos en una época de 
desigualdad. Nueva York: UNFPA. Recuperado de:

https://www.unfpa.org/sites/default/files/sowp/downloads/UNFPA_PUB_2017_ES

_SWOP_Estado_de_la_Poblacion_Mundial.pdf

United Nations/UN Department of Economic and Social Affairs (2017). World Population

Prospects: The 2017 Revision. Key Findings and Advance Tables.New York: UN.

Recuperado de:

https://esa.un.org/unpd/wpp/publications/files/wpp2017 keyfindings.pdf

United Nations/UN System Task Team on the Post-2015 UN Development Agenda (2012).

Sustainable Urbanization. Thematic Think Piece. UN Habitat. Recuperado de: http://www.un.org/en/development/desa/policy/untaskteam_undf/thinkpieces/18_ urbanization.pdf 